\title{
Relationship between limbal incisions and the structures of the anterior chamber angle
}

\author{
MOHAMED I. AYOUB AND AHMED H. SAID \\ Department of Ophthalmology, Faculty of Medicine, Cairo University, Egypt
}

It is essential for the ophthalmic surgeon who is incising the limbus to know exactly where his knife will penetrate the interior of the eye. Two factors must be taken into consideration:

(I) The external anatomical landmark in the limbal area.

(2) The angle made by the blade with the surface of the globe, i.e., the degree of obliquity of the incision. From the same external site an incision perpendicular to the scleral surface may penetrate the ciliary body, whereas a slanting incision parallel to the iris surface would enter the anterior chamber (Swan, I959).

Much controversy still exists concerning some of the external landmarks of the limbus, perhaps because of confusion between the conjunctival and the scleral overlap over the cornea. According to Wolff (rg68), the limbus is the transitional zone between the conjunctiva and the sclera on the one hand and the cornea on the other. The transe parent corneal tissue ends just behind a line which joins the ends of Bowman's membrane

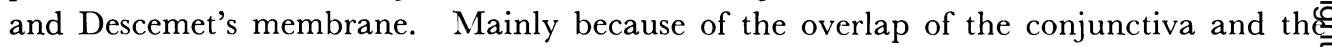
subconjunctival tissue, the former is I $\mathrm{mm}$. shorter than the latter, and Descemet's membrane also approaches nearer to the angle of the anterior chamber, although still separated from it by the whole of the corneo-scleral trabecular system, i.e. by about $\mathrm{I} \cdot 5 \mathrm{~mm}$. The oblique fibres and star-shaped cells characteristic of the sclera commence a little sooner anteriorly than posteriorly, but the main overlap of the cornea is by the conjunctiva and subconjunctival tissue rather than by the sclera as usually stated.

According to Stallard ( 1965 ), this scleral overlap is most extensive in the upper part of the limbus, less in the lower, and least in the horizontal meridian. Its extent shows individual variations, but accurate measurement of it is of prime importance in making an accurate incision into the apex of the filtration angle. The limits of the "surgical limbus", the apex of the filtration angle, may be judged with fair accuracy by using the well-known illumination phenomenon of "scleral scatter". To mark this site in the upper part of the globe, where most glaucoma operations are performed, a fine pencil of light is applied to the limbus at 6 o'clock. This illuminates a crescentic band about $2 \mathrm{~mm}$. wide, the scleral scatter, in the corneo-scleral zone. The apex of the filtration angle is then marked on the sclera at operation after reflecting the conjunctival flap.

Swan (I 959) stated that the limbus is not a sharp linear junction but a zone of transition which may be more than I $\mathrm{mm}$. wide. He commented that many authors, when referring to the limbus as a landmark, mean the junction of the limbal and corneal epithelium, i.e. the anterior border of the limbus. Swan defined the following landmarks:

(I) The anterior border of the limbus is the junction of the corneal and limbal epithelium. An incision made perpendicular to the surface of the globe at this border would be entirely 
in clear corneal stroma and would enter the anterior chamber through Descemet's membrane and the endothelium.

(2) The posterior border of the limbus appears as a relatively sharp line of demarcation between the bluish semitransparent limbal stroma and the opaque white sclera. Often this border cannot be seen through the conjunctiva and Tenon's capsule in the normal young adult. It is readily visible, however, in patients of all ages when the conjunctiva and Tenon's capsule are reflected. The posterior border of the limbus is difficult to recognize under the microscope. However, depending on microscopical identification of the scleral fibres, the same author concluded that, in most eyes, an incision made perpendicular to the surface of the stroma at this border will be entirely in avascular limbal stroma. It will enter the anterior chamber just in front of Schlemm's canal and just behind the posterior termination of Descemet's membrane and will pass through the anterior non-functional part of the trabecula. Swan believed this to be the ideal point of entry of incisions for iridectomy and cataract extraction.

The aim of this study is to clarify our knowledge of the relation of limbal incisions to internal structures by a more direct approach to the problem. Incisions were made in the limbal zone of cadaver eyes, which were then studied histologically to find out how they were related to the angle structures. In this manner the difficulty encountered in defining certain external landmarks on mere histological grounds was avoided.

\section{Material and methods}

Human eyes enucleated from cadavers within 4 hrs of death were kept at $4^{\circ} \mathrm{C}$. with the same precautions as those kept for corneal grafting. Before making the incisions, each eye was washed in normal saline and the 12 o'clock meridian was identified and marked. The condition of the cornea, the limbal landmarks, and the presence of healed trachomatous pannus (so common in our cases) were noted. The operating microscope was used $(\times 5)$ and the incision or incisions were made with a Bard Parker knife with blade No. 15. The incisions were all made perpendicular to the surface of the globe. Oblique incisions were avoided because the degree of inclination is more difficult to control, and because we believe that, if the surgeon has a clear mental picture of the site of penetration of a vertical incision, he can easily judge the site of penetration of oblique incisions.

In order to retain the regular shape of the eyeballs, it was not our intention in every case to penetrate the outer tunic of the eye but to induce deep scratches in the limbal area. The length of each incision was approximately $4 \mathrm{~mm}$. A black silk suture was placed in the sclera $2 \mathrm{~mm}$. posterior to the centre of each incision as a guide during the sectioning of paraffin blocks. The eyeballs were then fixed in formalin and paraffin sections were stained with haematoxylin and eosin.

The following incisions were studied:

(r) At the anterior border of the limbus (limbo-corneal):

(a) Without reflecting a conjunctival flap.

(b) After reflecting a conjunctival flap.

(2) At the posterior border of the limbus (limbo-scleral):

(a) Without reflecting a conjunctival flap.

(b) After reflecting a conjunctival flap.

(3) At the posterior limit of the scleral scatter.

In one series of eyeballs the above incisions were placed in the upper and/or lower limbus. In another series the incisions were placed nasally and/or temporally. Multiple incisions were some- 
times made in one eye. With this routine it was possible to study as many as sixty incisions in only 25 eyes.

To place an incision at the posterior margin of the scleral scatter, the following method was adopted. The conjunctiva was reflected in the area of the incision. To incise the upper limbus, focused light from a strong torch was used to illuminate the limbus at 6 o'clock. The torch was held almost parallel to the plane of the iris surface to obtain the scleral scatter at 12 o'clock. Using a needle dipped in gentian violet, the most posterior limit of the illuminated area was marked by fine dots of the dye. The light was then removed and a deep scratch was made connecting these marks. Measurements were made under the microscope by placing the histological preparations over a haemocytometer graticule.

\section{Results and discussion}

\section{(I) Incisions at the anterior border of the limbus (limbo-corneal)}

Whether the incision is made with the conjunctiva in place or after reflecting a conjunctival flap, it was always found to enter the anterior chamber well anterior to the termination of Descemet's membrane (Figs I, 3, and 4). This point of entry in the anterior chamber was found to be separated from the termination of Descemet's membrane by the following average distances: $\mathrm{I} \cdot 2 \mathrm{~mm}$. superiorly, $0.8 \mathrm{~mm}$. inferiorly, and $0.4 \mathrm{~mm}$. nasally and temporally. These distances naturally depend on the degree of conjunctival overlap, which is known to be widest above, less below, and least on the sides.

\section{(2) Incisions at the posterior border of the limbus (limbo-scleral)}

The limbo-scleral junction, i.e. the junction of the translucent limbal zone and the opaquen white sclera can easily be seen after reflecting a conjunctival flap. Although Swan (1959 holds that this border cannot be seen through the conjunctiva and Tenon's capsule in the normal young adult, we were able with the conjunctiva in place to define it as the junction between the very white sclera and the semitransparent limbal zone. In a number of eyes, after placing the incision in the limbo-scleral junction with the conjunctiva in place, the latter was excised to inspect the relation of the incision to the posterior limbal border. The relation was found to be correct in all cases.

Incisions placed at the posterior border of the limbus and made perpendicular to the surface of the globe were found to bear a constant relation to the internal structures. They entered the anterior chamber at or just anterior to the termination of Descemet's membrane. Such incisions do not cut into the trabecular meshwork and all the structures of the angle are posterior to the site of entry (Figs 2, 3, and 4). The point of entry into the anterior chamber was found to be separated from the centre of the lumen of Schlemm's canal by a distance of $0.45-0.55 \mathrm{~mm}$., a distance which does not vary in different meridia of the limbus. Our findings do not agree with those of Swan (1959), who believes that, in most eyes, these incisions enter the anterior chamber just in front of Schlemm's canal and just behind the termination of Descemet's membrane and pass through the non-functional part of the trabecula.

\section{(3) Incisions at the posterior limit of the scleral scatter}

Incisions placed perpendicular to the scleral surface at the posterior limit of the scleral scatter bear a constant relation to angle structures. Irrespective of the meridian, such incisions cut internally at a point in the sclera behind the scleral spur, i.e. they do not enter the anterior chamber but the supraciliary space about $0.5 \mathrm{~mm}$. behind the scleral spur (Figs 4 and 5). We found that the phenomenon of the scleral scatter cannot be taken as a 


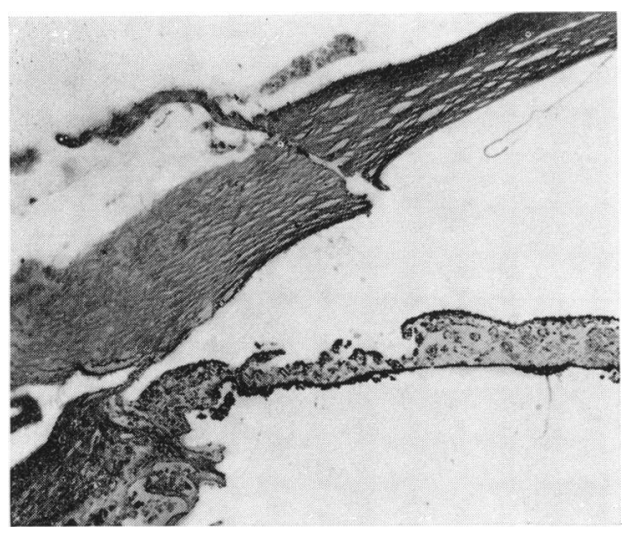

FIG I Incision at anterior border of upper limbus after reflection of conjunctiva. The anterior chamber is entered through Descemet's membrane (seen detached) away from the filtration angle. $\times 14$

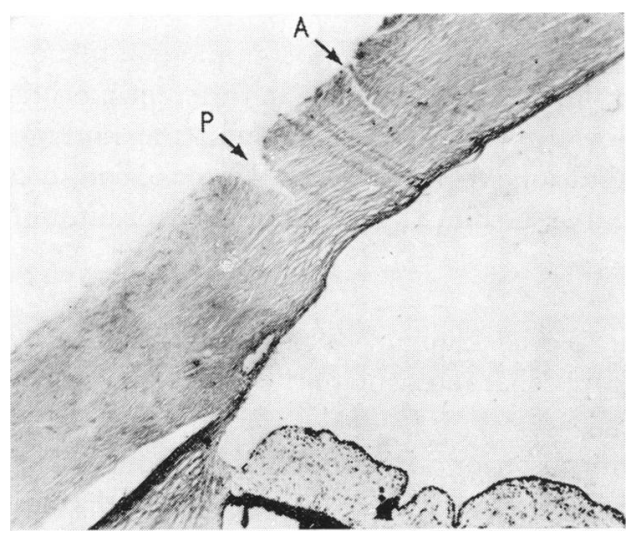

FIG 3 Two incisions at lower limbus

(A) At anterior border

(P) At posterior border.

The conjunctiva was excised. Note also the scleral overlap. $\times \mathrm{I} 4$

guide to the position of the apex of the filtration angle as advocated by Stallard (1965). For an incision started at the posterior end of the scleral scatter to enter the apex of the anterior chamber, the knife must be held obliquely to cut in a plane parallel to that of the iris. Even then it is possible to damage important angle structures (Fig. 6).

When two incisions were made in the same eye, one at the limbo-scleral junction and the other at the posterior limit of the scleral scatter (Fig. 4), the distance between the two was found to be between 0.85 and I $\cdot \mathrm{I} \mathrm{mm}$., showing that the breadth of the scatter is approximately $\mathrm{I} \mathrm{mm}$. behind the limbo-scleral junction.

We should like to stress the following observations concerning the conjunctival and scleral overlap:

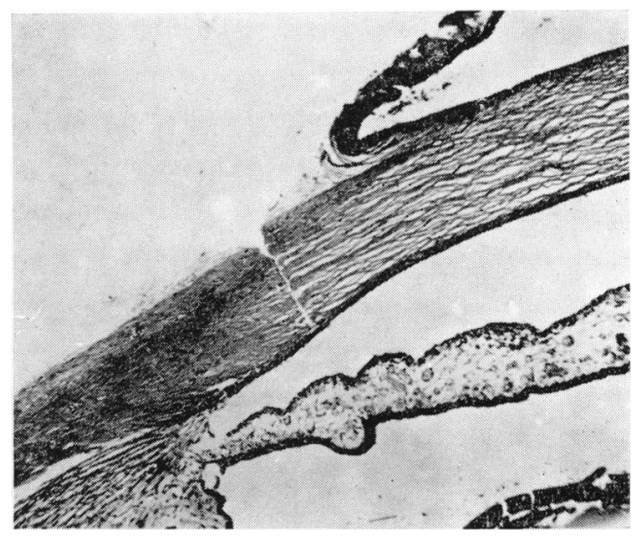

FIG 2 Incision at posterior border of upper limbus about to enter anterior chamber anterior to termination of Descemet's membrane. A conjunctival flap is reflected. The scleral fibres are seen to start sooner superficially, i.e. scleral overlap. $\times \mathrm{I} 4$

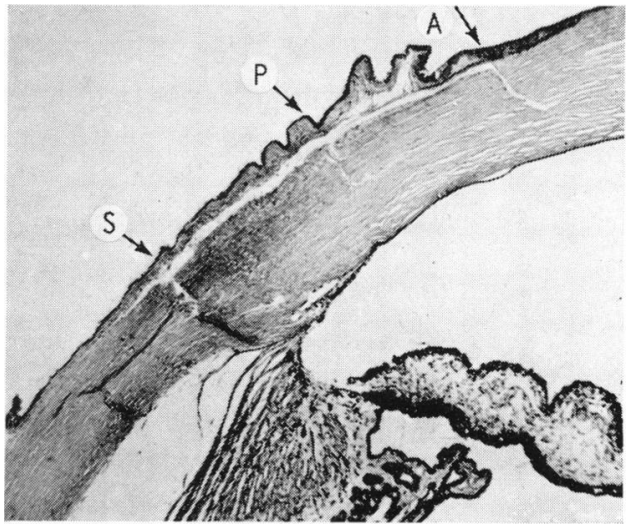

FIG 4 Three incisions placed at I2 o'clock

(A) At anterior border of limbus

(P) At posterior border

(S) At posterior limit of scleral scatter

None of the three incisions penetrated the globe. The scleral overlap is also seen. $\quad \times 14$ 


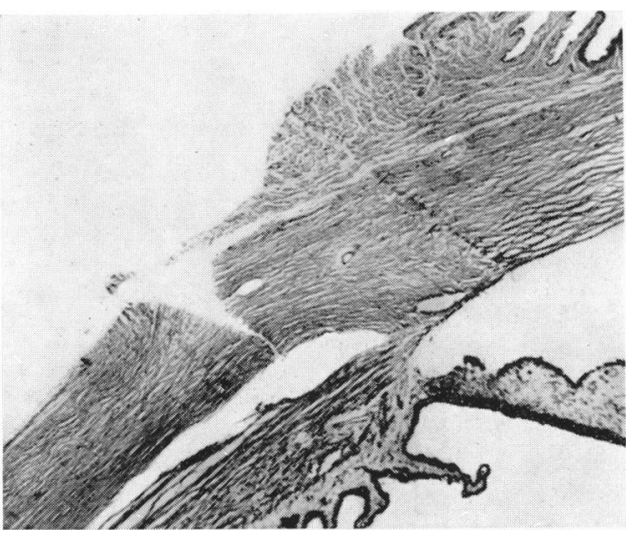

FIG 5 Incision placed at posterior end of zone of scleral scatter. It is seen to enter the supraciliary space. $\quad \times 14$

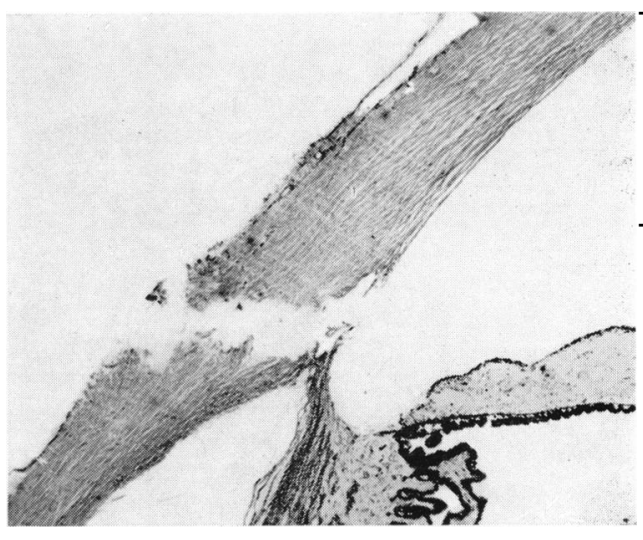

F IG 6 Incision started at posterior limit of scleral scatter and made in a plane parallel to the iris surface. Schlemm's canal and the trabecular meshwork are grossly damaged. $\quad \times 14$

(I) The conjunctival overlap

The main overlap of the cornea is by conjunctiva and subconjunctival tissue. The degree $\bigcirc$ of this overlap varies in different eyes and in the same eye in different meridia. It is most $\frac{\rho}{0}$ marked above $(1.2 \mathrm{~mm}$.), less below $(0.8 \mathrm{~mm}$.), and least in the horizontal meridian $\stackrel{\mathbb{D}}{工}$ $(0 \cdot 4 \mathrm{~mm}$.). The breadth of the limbal zone depends on the degree of conjunctival overlam $\vec{\varphi}$ since the anterior border of the limbal area coincides with the anterior limit of this overlat

(2) The scleral overlap

That the sclera overlaps the coruea is well known. The overlap can easily be detected microscopically, since the oblique fibres characteristic of the sclera are seen to start sooner superficially (Figs 2, 3, and 4).

From the surgical point of view, the posterior border of the limbus represents the anterior limit of the scleral overlap. Since we found that incisions placed at the posterior border of the limbus are always related in the same way to the internal structures, we believe that the scleral overlap, unlike the conjunctival overlap, is of the same degree in all meridians.

\section{Conclusion}

From the surgical point of view, the limbus is a translucent transitional zone between the clear cornea and the white sclera, and its breadth averages $\mathrm{I} \cdot 2 \mathrm{~mm}$. superiorly, $0.8 \mathrm{~mm}$. inferiorly, and $0.4 \mathrm{~mm}$. nasally and temporally. The corneal periphery is overlapped by $\frac{9}{5}$ the conjunctiva and sclera. The conjunctival overlap is most evident above and its extent $\frac{D}{0}$ determines the breadth of the limbal zone. It can be reflected by blunt dissection. The scleral overlap can be seen under the microscope. The posterior border of the limbus of represents its superficial (anterior) limit. Unlike the conjunctival overlap, its extent is the $\mathbb{N}$ same all round the cornea.

The anterior border of the limbus can be defined as the junction of the transparent cornea with the semi-transparent limbal zone. It corresponds to the apex of the conjunctival $\stackrel{\varrho}{\simeq}$ overlap and the termination of Bowman's membrane. After a conjunctival incision, $\stackrel{\mathbb{\infty}}{\rightarrow}$ it corresponds to the most anterior limit to which the conjunctiva can be reflected. An incision placed at this border will enter the anterior chamber through Descemet's membrane 
and will be separated from its termination (ring of Schwalbe) by the average distances of $\mathrm{I} \cdot 2 \mathrm{~mm}$. superiorly, $0.8 \mathrm{~mm}$. inferiorly, and $0.4 \mathrm{~mm}$. nasally and temporally. It is a less valuable surgical landmark than the posterior border of the limbus for the following reasons:

(I) The degree of conjunctival overlap varies in different persons and in different meridia.

(2) After a conjunctival incision, its situation is often influenced by the degree and ease of dissection.

(3) When trachomatous pannus or other opacities involve the corneal periphery, the definition of the anterior border of the limbus is often lost.

The posterior border of the limbus is the junction of the translucent limbal zone with the white sclera. It is a sharp line which can be made out before and naturally more easily after reflecting the conjunctiva. It is the most reliable surgical landmark when its relation to internal structures are considered. An incision made perpendicular to the surface of the globe at this border enters the anterior chamber at or just anterior to the termination of Descemet's membrane and approximately $0.5 \mathrm{~mm}$. from the centre of the lumen of Schlemm's canal, i.e. all the structures of the filtration angle are behind it. This relation is constant all round the cornea.

The phenomenon of scleral scatter illuminates (besides the zone of the limbus) a zone approximately I $\mathrm{mm}$. behind the posterior border of the limbus. A perpendicular incision placed at the posterior limit of the scatter, will enter the supraciliary space approximately $0.5 \mathrm{~mm}$. behind the scleral spur.

\section{Summary}

Using cadaver eyes, incisions were made in the different external landmarks of the limbus in different meridians. Such eyes were then subjected to histological examination to study the relation of these incisions to the structures of the angle of the anterior chamber.

\section{References}

STAllard, H. в. (1965) "Eye Surgery", 4th ed., pp. 634-635. Wright, Bristol

swAN, K. C. (1959) "Surgical Anatomy in Relation to Glaucoma" in "Symposium on Glaucoma", ed. W. B. Clark, pp. $3^{8-52 . ~ M o s b y, ~ S t . ~ L o u i s ~}$

WOLFF, E. (1968) "Eugene Wolff's Anatomy of the Eye and Orbit", 6th ed., revised by R. J. Last.

Lewis, London 